\title{
Reactive oxygen species mediate angiotensin II-induced transcytosis of low-density lipoprotein across endothelial cells
}

\author{
FANG BIAN $^{1,2}$, JUN CUI ${ }^{3}$, TAO ZHENG ${ }^{2,4}$ and SI JIN ${ }^{2}$ \\ ${ }^{1}$ Department of Pharmacy, The Affiliated Hospital of Xiangyang Central Hospital of Hubei University of Arts and Science, \\ Xiangyang, Hubei 441000; ${ }^{2}$ Department of Endocrinology, Institute of Geriatric Medicine, Liyuan Hospital, \\ Tongji Medical College, Huazhong University of Science and Technology, Wuhan, Hubei 430030; \\ ${ }^{3}$ Department of Cardiothoracic Surgery, The Affiliated Hospital of Xiangyang Central Hospital \\ of Hubei University of Arts and Science, Xiangyang, Hubei 441000; ${ }^{4}$ Department of Pharmacy, \\ Taihe Hospital, Hubei University of Medicine, Shiyan, Hubei 442000, P.R. China
}

Received March 22, 2016; Accepted January 3, 2017

DOI: $10.3892 / \mathrm{ijmm} .2017 .2887$

\begin{abstract}
The retention of plasma low-density lipoprotein (LDL) particles to subendothelial spaces through transcytosis across the endothelium is the initial step of atherosclerosis (AS). Angiotensin II (Ang II), as the principal effector molecule of the renin-angiotensin system (RAS), is implicated in several important steps of AS development. However, whether or not Ang II can directly exert a pro-atherogenic effect by promoting LDL transcytosis across endothelial barriers, has not been defined. In the present study, we found that Ang II upregulated intracellular reactive oxygen species (ROS) levels in endothelial cells (ECs) by measuring fluorescence of 2',7'-dichlorofluorescein (DCF-DA). Based on our transcytosis model, we observed that Ang II significantly accelerated LDL transcytosis, whereas transcytosis inhibitor methyl- $\beta$-cyclodextrin $(\mathrm{M} \beta \mathrm{CD})$ and ROS inhibitor dithiothreitol (DTT), markedly blocked the Ang II-stimulated increase in LDL transcytosis. Confocal imaging analysis revealed that both LDL uptake by cells and LDL retention in human umbilical venous walls were highly elevated after Ang II exposure, while M $\beta C D$ and DTT significantly inhibited the effects of Ang II. What is more, proteins involved in caveolae-mediated transcytosis, including LDL receptor (LDLR), caveolin-1 and cavin-1, were associated with Ang II-induced LDL transcytosis across the ECs. Nevertheless, this process was independent of clathrin in our study. Of note, ROS inhibitor, DTT, markedly decreased the expression levels of those proteins. Consequently, ROS are critical mediators in Ang II-induced LDL transcytosis. Hopefully, these findings
\end{abstract}

Correspondence to: Professor Si Jin, Department of Endocrinology, Institute of Geriatric Medicine, Liyuan Hospital, Tongji Medical College, Huazhong University of Science and Technology, Wuhan, Hubei 430077, P.R. China

E-mail: jinsi@mail.hust.edu.cn

Key words: atherosclerosis, low-density lipoprotein, transcytosis, angiotensin II, reactive oxygen species, caveolae will provide novel insight into the crosstalk between dyslipidemia and RAS in atherogenesis.

\section{Introduction}

Atherosclerosis (AS) is the predominant pathological basis of cardiovascular disease, and its prevalence worldwide has reached epidemic proportions. Current evidence suggests that the subendothelial retention of apoB100 containing lipoproteins (e.g., low-density lipoprotein, LDL) is the initial step of atherogenesis, which is usually termed the 'response to retention' hypothesis in the literature (1-4). Since the diameter of LDL particles $(20-30 \mathrm{~nm})$ is much larger than the gap junctions (3-6 nm) between vascular endothelial cells (ECs), the only pathway for LDL particles to traffic across the intact endothelial barrier is through a transporting process termed 'transcytosis', which was postulated to describe the transport of macromolecular cargo from one side of polar cells to the other within membrane-bound carriers $(5,6)$. Notably, many studies have pointed to a possible link between LDL transcytosis across ECs and the initiation of AS $(2,6,7)$.

There is increasing evidence of the tight interactions between AS development and renin-angiotensin system (RAS) activation. A meta-analysis reported that Ang-converting enzyme (ACE) inhibitors or Ang receptor blockers (ARBs) are beneficial in normotensive (systolic pressure $<130 \mathrm{mmHg}$ ) atherosclerotic patients (8). As we know, ACE plays a key role in RAS as it converts angiotensin I (Ang I) to Ang II. Furthermore, ACE is abundant in vulnerable lesions and is expressed in macrophage foam cells, the ECs of neovessels, as well as smooth muscle cells (SMCs) $(9,10)$.

Moreover, as the principal effector molecule of RAS, Ang II is implicated in several important steps of AS, such as vascular inflammation, vascular remodeling, thrombosis, and plaque rupture. Ang II has been shown to accelerate AS by activating various signaling pathways and augmenting oxidative stress (11-14). In injured arteries, Ang II provides a positive feedback loop for vascular inflammation by recruiting inflammatory cells, which then generate more 
Ang II, therefore perpetuating vascular inflammation (15). It has been suggested that Ang II upregulates the levels of proinflammatory cytokines [interleukin (IL)-6, monocyte chemoattractant protein-1 (MCP-1), vascular cell adhesion molecule-1 (VCAM-1)] via type 1 Ang II receptor $\left(\mathrm{AT}_{1} \mathrm{R}\right)$ and activates the nuclear factor- $\kappa \mathrm{B}(\mathrm{NF}-\kappa \mathrm{B})$ signaling pathway, which would deteriorate the atherosclerotic inflammation response (16-19).

Ang II-induced reactive oxygen species (ROS)-mediated DNA damage may also aggravate the progression of AS. Indeed, evidence indicates that Ang II is able to induce the production of superoxide anions and activate the NADH/NADPH signaling pathway (20). Meanwhile, data have revealed that $\mathrm{AT}_{1 \mathrm{~A}} \mathrm{R}$ deficiency-induced reduction of oxidative stress, apoptosis, and matrix metalloproteinase expression in atherosclerotic lesions may decrease plaque size (21). Interestingly, Daugherty et al found that $\mathrm{AT}_{1 \mathrm{~A}} \mathrm{R}$ deficiency-induced reduction in AS was independent of systolic blood pressure, oxidation and chemoattractants. They confirmed that hypercholesterolemia-induced Ang peptide production provides a basis for $\mathrm{AT}_{1 \mathrm{~A}} \mathrm{R}$ deficiency-induced reductions in AS (22).

In addition, AS is considered to be associated with premature biological aging. Kunieda et al found that Ang II promotes vascular inflammation and augments AS by inducing premature senescence of SMCs via a p21-dependent pathway (23). Wang et al suggested that whole body receptor-associated protein (RAP) deficiency could attenuate the incidence of AS in hypercholesterolemic mice infused with Ang II (24). In addition, evidence demonstrated that increased local RAS production leads to the pathophysiological process of vascular remodeling, which is an important event in the progression of AS $(25,26)$. Some studies reported that Ang II mediates the expression of focal adhesion kinases and integrins in cardiac fibroblasts, the levels of c-fos, EGFR1, transforming growth factor- $\beta$ and extracellular matrix proteins in cardiac myocytes as well as SMC proliferation and hypertrophy $(27,28)$.

It should also be noted that the crosstalk between dyslipidemia and RAS activation in atherogenesis has been suggested. Previous research revealed that Ang II infusion accelerated an increased severity of aortic atherosclerotic lesions and aneurysms in apoE $E^{-/-}$mice (11). A recent study revealed that Ang II infusion exaggerated AS development in apoE $\mathrm{E}^{-/-}$mice by enhancing the accumulation of dihydroethidium-positive mononuclear cells in the intima and mRNA expression levels of Nox2 (29). Native or oxidized low-density lipoprotein (ox-LDL) has previously been reported to enhance the levels of $\mathrm{ACE}$ and $\mathrm{AT}_{1 \mathrm{~A}} \mathrm{R}$ in human ECs and SMCs through LDL receptors (LDLRs) or lectin-like ox-LDL receptor-1 (LOX-1), respectively (30). In addition, Ang II may facilitate LDL oxidation and uptake, as well as thrombosis (31). It was shown that Ang II infusion stimulated a significant increase in aortic LDL receptor-related protein (LRP1) expression and lipid infiltration in the arterial intima (32). Previously, evidence indicates that LDL uptake by rat aorta is increased by Ang II, whereas this effect may be independent of its pressor action (33). Moreover, Keidar et al observed that the mechanism of Ang II atherogenicity is associated with its effect on the uptake of ox-LDL by macrophage and foam cell formation, a process mediated by IL-6 (34).
In our previous study, we demonstrated that C-reactive protein (CRP) promotes AS by directly increasing the transcytosis of LDL across ECs and accelerating LDL retention in vascular walls, which is mediated by ROS (2). Moreover, tumor necrosis factor- $\alpha$ (TNF- $\alpha$ ) was also proven to promote early AS by upregulating LDL transcytosis and LDL retention in vascular walls (3). However, whether or not Ang II is able to directly exert a pro-atherogenic effect by promoting LDL transcytosis across the endothelial barrier and LDL retention in vascular walls, has not been defined.

In the present study, based on the in vitro model to assay the transcytosis of LDL, we found that Ang II indeed increased LDL transcytosis across ECs and accelerated LDL retention in the subendothelial space of human umbilical venous walls. Following this, we further explored the underlying molecular mechanisms and revealed that the upregulation of transcytosisrelated proteins is involved in Ang II-induced LDL transcytosis, which is associated with the production of ROS.

\section{Materials and methods}

The present study was approved by the Ethics Committee of Tongji Medical College, Huazhong University of Science and Technology (Wuhan, China) and conducted in accordance with the Declaration of Helsinki and all applicable national and local regulations. All subjects provided written informed consent prior to the initiation of the study.

Reagents and chemicals. Endothelial cell medium (ECM) (cat. no. 1001) with $10 \%$ fetal bovine serum (FBS), $100 \mathrm{U} / \mathrm{ml}$ penicillin, $100 \mathrm{U} / \mathrm{ml}$ streptomycin and endothelial cell growth supplement (ECGS) were purchased all from ScienCell Research Laboratories (Carlsbad, CA, USA). Fluorescein isothiocyanate (FITC) (cat. no. FD150S) was purchased from Sigma-Aldrich (St. Louis, MO, USA). LDL (cat. no. YB-001) was obtained from Yiyuan Biotechnology Co., Ltd. (Guangzhou, China). 2',7'-Dichlorofluorescein (DCF-DA) was obtained from Applygen Technologies, Inc. (Beijing, China). BCA assay kit (cat. no. 23235) was obtained from Thermo Fisher Scientific, Inc. (Rockford, IL, USA). Ang II and methyl- $\beta$-cyclodextrin (M $\beta C D)$ were supplied by Sigma-Aldrich. Dithiothreitol (DTT) was purchased from MDBio, Inc. (Piscataway, NJ, USA). RIPA lysis buffer (cat. no. AR0105) was from Beyotime Institute of Biotechnology (Shanghai, China). Caveolin-1 (cat. no. 3238) was obtained from Cell Signaling Technology, Inc. (Danvers, MA, USA). Cavin-1 (cat. no. 18892), LDLR (cat. no. 10785), clathrin (cat. no. 10852) and $\beta$-actin (cat. no. 14395) were supplied by Proteintech Group, Inc. (Chicago, IL, USA).

Isolation and culture of human umbilical vein endothelial cells (HUVECs). Isolation of HUVECs was performed as described in our previous study (2). HUVECs were cultured in ECM with $10 \% \mathrm{FBS}, 100 \mathrm{U} / \mathrm{ml}$ penicillin, $100 \mathrm{U} / \mathrm{ml}$ streptomycin and $30 \mu \mathrm{g} / \mathrm{ml} \mathrm{ECGS}$ at $37^{\circ} \mathrm{C}$ with $5 \% \mathrm{CO}_{2}$. Cells were passaged when $80-90 \%$ confluent and were used between passages 3 and 9 .

LDL labeling. LDL was labeled using FITC as described in our previous study (2). In brief, $2 \mathrm{mg}$ LDL and $120 \mu \mathrm{g}$ FITC were 
mixed and incubated in the dark $\left(37^{\circ} \mathrm{C}, 2 \mathrm{~h}\right)$. Unbound FITC was removed by dialysis against $\mathrm{PBS}$ in the dark $\left(4^{\circ} \mathrm{C}, 72 \mathrm{~h}\right)$. After the measurement of protein with the BCA assay kit, FITC-LDL was then stored at $4^{\circ} \mathrm{C}$ in the dark for further use.

Measurements of intracellular ROS levels. For determining the intracellular levels of ROS, HUVECs were incubated with $3 \mu \mathrm{mol} / 1 \mathrm{DCF}-\mathrm{DA}$ at room temperature in the dark for $30 \mathrm{~min}$ as previously described (2), and then the medium with DCF was removed. The cells were pretreated with $30 \mu \mathrm{mol} / 1 \mathrm{DTT}$ for a further $30 \mathrm{~min}$, followed by exposure to $10^{-9} \mathrm{M}$ Ang II for $30 \mathrm{~min}$. Using a fluorescence spectrophotometer (Infinite F200 PRO; Tecan, Männedorf, Switzerland) with excitation and emission wavelengths of 490 and $520 \mathrm{~nm}$, respectively, the fluorescence intensity of the ROS-reactive dichlorofluorescin was dynamically monitored.

Analysis of the transcytosis of LDL across HUVECs using an in vitro transcytosis model. LDL transcytosis across HUVECs was determined based on the newly established transcytosis model established in our laboratory. As shown in Fig. 1, cells were seeded $\left(\sim 4 \times 10^{4}\right.$ cells/insert $)$ on a polyester membrane of a Costar Transwell (6.5-mm diameter, $0.4-\mu \mathrm{m}$ pore size) (Corning Costar, Cambridge, MA, USA) to form an integrated cell monolayer. As depicted in a previous study (2), two inserts of cell monolayers with equal integrity were divided into the same group: the control insert and the naive insert, respectively. The control insert was stimulated with FITC-LDL to determine the total amount of LDL transport. Paracellular transport was analyzed by treatment with FITC-LDL and 6 -fold excess of unlabeled LDL in the naive insert. Thereafter, the amount of LDL transcytosis was calculated by subtracting the paracellular transport (the competitive insert) from the total transport (the non-competitive insert). In the present study, following pretreatment with 3,000 $\mu \mathrm{M} \mathrm{M} \beta \mathrm{CD}$ or $30 \mu \mathrm{mol} / 1 \mathrm{DTT}$ for $30 \mathrm{~min}$, HUVECs were then stimulated with $50 \mu \mathrm{g} / \mathrm{ml}$ FITC-LDL (and/or LDL) and $10^{-9} \mathrm{M}$ Ang II for $24 \mathrm{~h}$. After $24 \mathrm{~h}$, the samples were collected from the outer chambers and further dialyzed against PBS to remove the free FITC. The relative fluorescence was measured via a fluorescence spectrophotometer with excitation and emission wavelengths of 490 and $520 \mathrm{~nm}$, respectively. The amount of LDL transcytosis was normalized to that obtained in the control group.

Confocal imaging analysis of the uptake of LDL in HUVECs. Cells were seeded on gelatin-coated glass coverslips in 24-well culture plates $\left(37^{\circ} \mathrm{C}, 5 \% \mathrm{CO}_{2}\right)$. To determine LDL uptake in the HUVECs, the cells were first incubated with $50 \mu \mathrm{g} / \mathrm{ml}$ FITC-LDL for $24 \mathrm{~h}$ and then treated with or without $10^{-9} \mathrm{M}$ Ang II, 3,000 $\mu \mathrm{M} \mathrm{M} \beta \mathrm{CD}$ or $30 \mu \mathrm{mol} / 1 \mathrm{DTT}$ for $24 \mathrm{~h}$ at $37^{\circ} \mathrm{C}$. Images were obtained by confocal laser scanning microscopy (Olympus FV500; Olympus, Center Valley, PA, USA) (the excitation wavelength, $490 \mathrm{~nm}$; the emission wavelength, $520 \mathrm{~nm}$ ). The fluorescence images were analyzed using ImageJ software. Each individual microscopic field was randomly selected to include at least 15 cells, and the numbers of cells were counted. The integrated fluorescence intensities were measured and normalized to the number of cells.
Confocal imaging analysis of $L D L$ retention in the isolated human umbilical venous wall. The human umbilical venous rings were bubbled with a mixed gas $\left(95 \% \mathrm{O}_{2}, 5 \% \mathrm{CO}_{2}\right)$ and incubated with $50 \mu \mathrm{g} / \mathrm{ml}$ FITC-LDL, and/or $10^{-9} \mathrm{M}$ Ang II, $3,000 \mu \mathrm{M} \mathrm{M} \beta \mathrm{CD}$ or $30 \mu \mathrm{mol} / 1 \mathrm{DTT}$ for $3 \mathrm{~h}$ at $37^{\circ} \mathrm{C}$. Then, all tissues were frozen and consecutively cut into sections of $10-\mu \mathrm{m}$ in thickness with a cryostat (Leica CM1900; Leica Microsystems, Wetzlar, Germany), and further stained with DAPI. For the fluorescence quantification, we used a weighted protocol described previously (2). The region above the basilar membrane was defined as the region of interest (ROI). The fluorescent area and the area of ROI were quantified using ImageJ software. As previously described (2), the weighted analysis was performed by first determining the area of fluorescence within the ROI of each optical section for three fluorescence intensity value ranges: 86 to 123,124 to 161 , and 162 to 200 . These three area measurements were then multiplied by 1,2 or 3, respectively, to give greater weight to areas of highest intensities. These weighted values were then summed for each optical section and divided by the area of the ROI.

Western blotting. HUVECs were seeded in 100-mm culture dishes. Following pretreatment with 3,000 $\mu \mathrm{M} \mathrm{M} \beta \mathrm{CD}$ or $30 \mu \mathrm{mol} / 1$ DTT for $30 \mathrm{~min}$, the HUVECs were then stimulated with or without $10^{-9} \mathrm{M}$ Ang II for $24 \mathrm{~h}$. Cells were lysed with the RIPA lysis buffer. Resuspended proteins were separated by SDS-PAGE gel and transferred to a PVDF membrane. The membranes were probed with primary antibodies against LDLR (1:700), caveolin-1 (1:8,000), cavin-1 $(1: 5,000)$, clathrin $(1: 1,000)$ and $\beta$-actin $(1: 4,000)$. The immunoreactive bands were visualized by an ECL western blot detection system. The expression of proteins was normalized to the control group.

Statistical analysis. All data are expressed as the mean \pm SEM from at least three separate experiments. SPSS 13.0 software (SPSS, Inc., Chicago, IL, USA) was used for all statistical analysis. Student's unpaired t-test was used to analyze individual group statistical comparisons, and one-way ANOVA with post-hoc test was performed to evaluate multiple group comparisons. Statistical significance is defined as $\mathrm{P}<0.05$.

\section{Results}

ROS are involved in Ang II-induced LDL transcytosis across HUVECs. In this study, we ascertained whether Ang II could promote LDL transcytosis across HUVECs based on the established transcytosis model. The amount of LDL transcytosis was normalized to that obtained in the control group. As shown in Fig. 2A, Ang II exposure for $24 \mathrm{~h}$ significantly elevated the level of LDL transcytosis. Transcytosis inhibitor, M $\beta C D$, highly attenuated Ang II-induced LDL transcytosis. Ang II increased intracellular ROS levels, in agreement with previous studies. ROS inhibitor, DTT, markedly decreased the level of ROS induced by Ang II (Fig. 2B). In a previous study, we demonstrated that $\mathrm{H}_{2} \mathrm{O}_{2}$ was able to increase LDL transcytosis (2). In this study, ROS inhibitor, DTT, significantly decreased LDL transcytosis induced by Ang II, as depicted in Fig. 2A. 


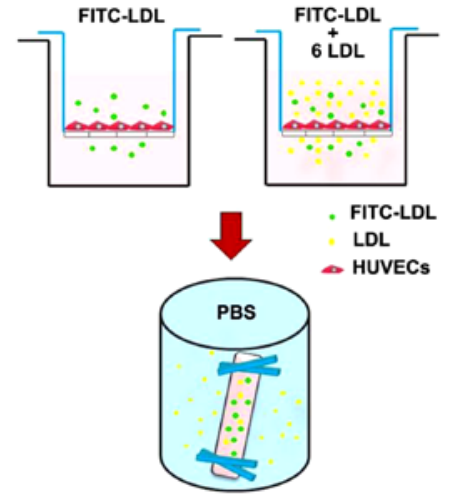

Figure 1. The established in vitro low-density lipoprotein (LDL) transcytosis model. Cells were seeded $\left(\sim 4 \times 10^{4}\right.$ cells/insert) on a polyester membrane of a Costar Transwell (6.5-mm diameter, $0.4-\mu \mathrm{m}$ pore size) to form an integrated cell monolayer. Two inserts of cell monolayers with equal integrity were divided into the same group: the control insert and the naive insert, respectively. The control insert was stimulated with fluorescein isothiocyanate (FITC)-LDL to determine the total amount of LDL transport. Paracellular transport was analyzed by treatment with FITC-LDL and 6-fold excess of unlabeled LDL in the naive insert. After treatment, samples were collected from the outer chambers and further dialyzed against PBS to remove the free FITC. The relative fluorescence was measured via a fluorescence spectrophotometer.
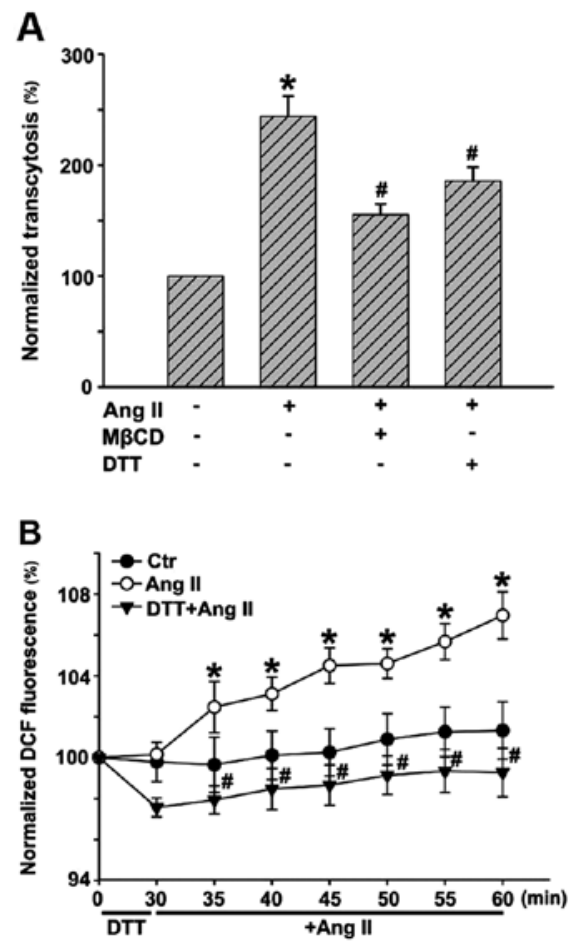

Figure 2. (A) Reactive oxygen species (ROS) are involved in angiotensin II (Ang II)-induced low-density lipoprotein (LDL) transcytosis across human umbilical vein endothelial cells (HUVECs). HUVECs were pretreated with methyl- $\beta$-cyclodextrin $(\mathrm{M} \beta \mathrm{CD}$ ) or dithiothreitol (DTT) for $30 \mathrm{~min}$, and then stimulated with fluorescein isothiocyanate (FITC)-LDL (and/or LDL) and Ang II for $24 \mathrm{~h}$. After $24 \mathrm{~h}$, samples were collected from the outer chambers and further dialyzed against PBS to remove the free FITC. (B) The relative fluorescence was measured via a fluorescence spectrophotometer. The amount of LDL transcytosis was normalized to that obtained in the control group. ${ }^{*} \mathrm{P}<0.05$ vs. control; ${ }^{\text {}} \mathrm{P}<0.05$ vs. Ang II.

Ang II stimulation increases the uptake of LDL in HUVECs. After incubation with FITC-LDL, the HUVECs were found
A
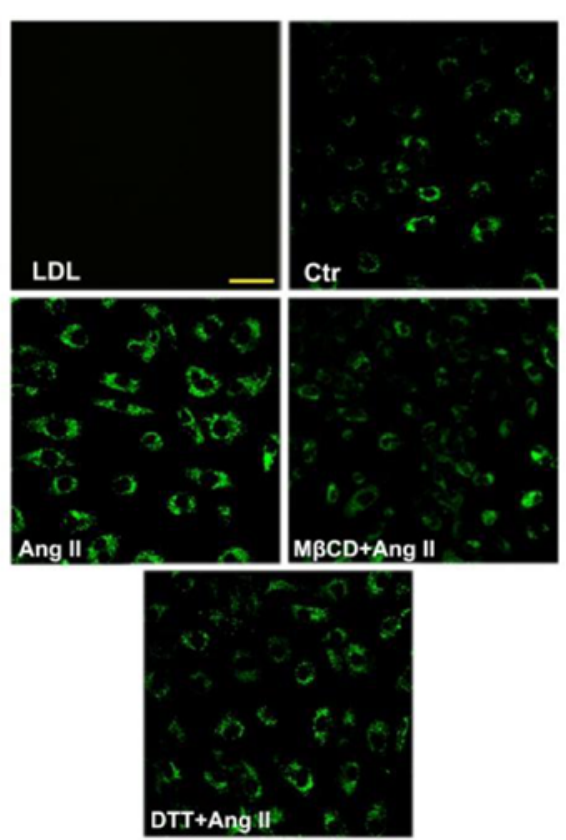

B

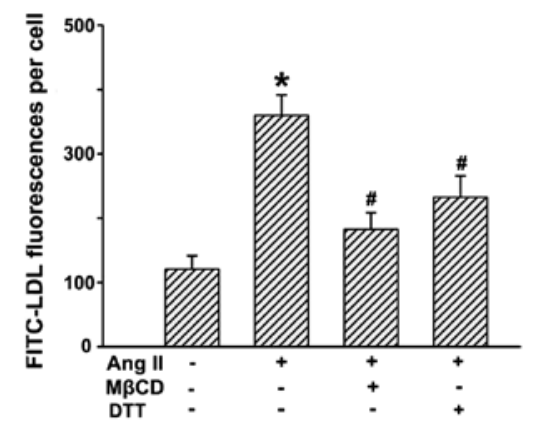

Figure 3. Angiotensin II (Ang II) stimulation increases the uptake of low-density lipoprotein (LDL) in human umbilical vein endothelial cells (HUVECs). HUVECs were first incubated with fluorescein isothiocyanate (FITC)-LDL for $24 \mathrm{~h}$ and then treated with or without Ang II, methyl- $\beta$-cyclodextrin (M $\beta C D$ ), and dithiothreitol (DTT) for $24 \mathrm{~h}$ at $37^{\circ} \mathrm{C}$. (A) Confocal microscopy images of FITC-LDL uptake stimulated by Ang II alone or after pretreatment with $\mathrm{M} \beta C \mathrm{C} / \mathrm{DTT}$ in HUVECs. Scale bars, $50 \mu \mathrm{m}$. (B) Quantification summary of FITC-LDL uptake in HUVECs. ${ }^{*} \mathrm{P}<0.05$ vs. control; ${ }^{*} \mathrm{P}<0.05$ vs. Ang II.

to be full of small, individual, discrete vesicles throughout the cells. The fluorescent intensity in each individual cell reflected the extent of LDL uptake. Since LDL uptake is an intermediate phase of LDL transcytosis, it may also represent the amount of LDL transcytosis to a certain degree. As shown in Fig. 3, Ang II significantly enhanced fluorescence intensities in the cells, which indicated an increase in LDL uptake. In contrast, M $\beta C D$ and DTT markedly inhibited Ang II-stimulated LDL internalization

Ang II stimulation increases the retention of $L D L$ in human umbilical venous walls. Subendothelial retention of apoB-containing lipoprotein particles (e.g., LDL) is important for the initiation of AS. An experiment was conducted to demonstrate whether Ang II could promote subendothelial retention of LDL in human umbilical venous walls. As depicted in Fig. 4, only a small amount of LDL was determined in human umbilical venous walls after incubation with FITC-LDL for $24 \mathrm{~h}$, while much more FITC-LDL accumulated in the 

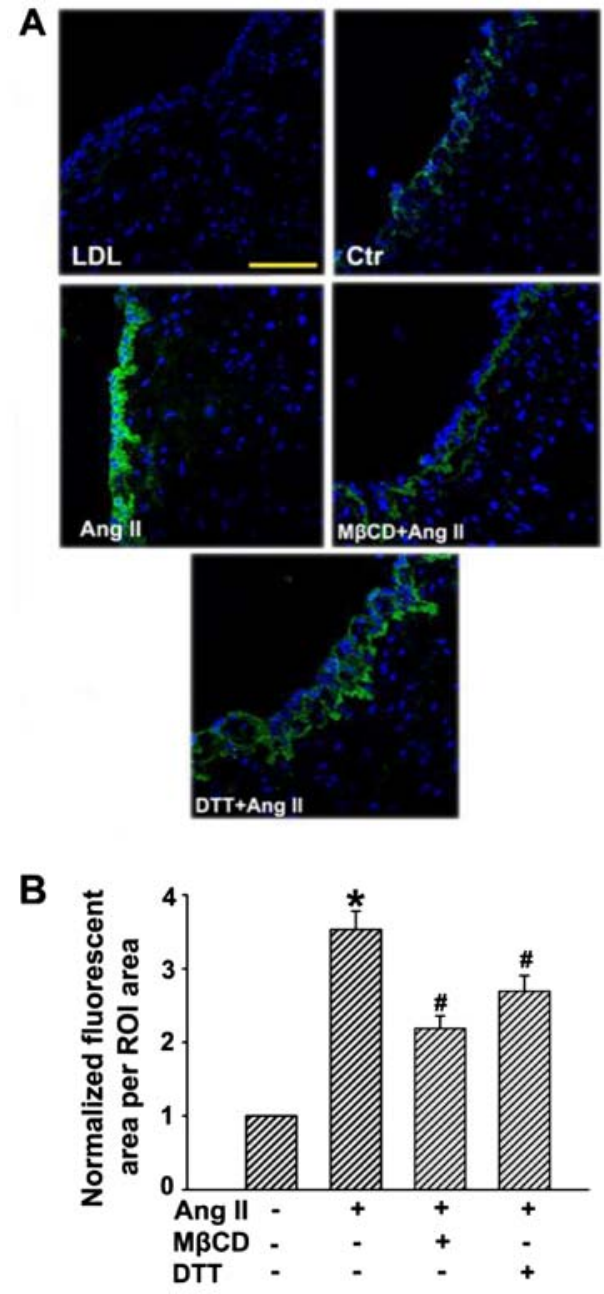

Figure 4. Angiotensin II (Ang II) stimulation increases the retention of low-density lipoprotein (LDL) in human umbilical venous walls. The human umbilical venous rings were bubbled with a mixed gas $\left(95 \% \mathrm{O}_{2}, 5 \% \mathrm{CO}_{2}\right)$ and incubated with fluorescein isothiocyanate (FITC)-LDL, and/or Ang II, methyl- $\beta$-cyclodextrin $\left(\mathrm{M} \beta \mathrm{CD}\right.$ ), dithiothreitol (DTT) for $3 \mathrm{~h}$ at $37^{\circ} \mathrm{C}$. (A) Confocal microscopy images of FITC-LDL retention stimulated by Ang II alone or after pretreatment with $\mathrm{M} \beta \mathrm{CD} / \mathrm{DTT}$ in human umbilical venous rings. Scale bars, $100 \mu \mathrm{m}$. (B) Quantification summary of FITC-LDL retention in vessels. ${ }^{*} \mathrm{P}<0.05$ vs. control; ${ }^{\#} \mathrm{P}<0.05$ vs. Ang II.

region above the basilar membrane after Ang II stimulation. However, the retention of FITC-LDL was markedly inhibited in the presence of $\mathrm{M} \beta \mathrm{CD}$ or DTT.

Ang II stimulates the expression of molecules involved in LDL transcytosis. As shown in Fig. 5, Ang II stimulation for $24 \mathrm{~h}$ distinctly upregulated the expression of LDLR, caveolin-1 and cavin-1, while it had no obvious effect on the level of clathrin. Pretreatment with M $\beta C D$ or DTT for $30 \mathrm{~min}$ significantly blocked Ang II-induced upregulation of these proteins. Thus, Ang II-stimulated LDL transcytosis may be partly due to the elevated expression of the proteins involved in caveolae-mediated transcytosis. Of note, ROS are critical factors in Ang II-induced LDL transcytosis.

\section{Discussion}

Currently, it is generally accepted that the subendothelial retention of apoB-containing lipoprotein particles (e.g., LDL)

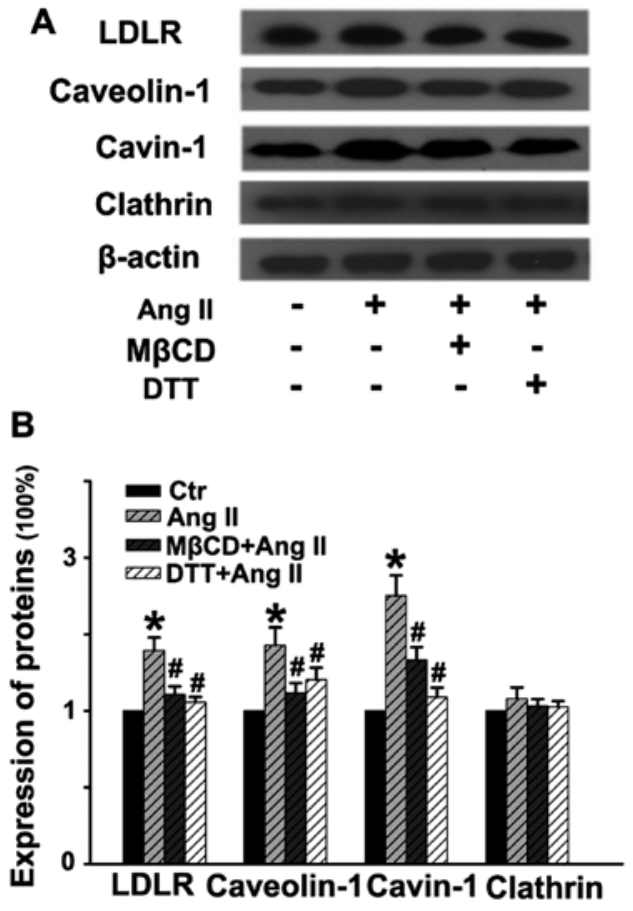

Figure 5. Angiotensin II (Ang II) stimulates the expression of molecules involved in low-density lipoprotein (LDL) transcytosis. Human umbilical vein endothelial cells (HUVECs) were pretreated with methyl- $\beta$-cyclodextrin (M $\beta C D$ ) or dithiothreitol (DTT) for $30 \mathrm{~min}$, and then stimulated with or without Ang II for $24 \mathrm{~h}$. (A) Representative western blots showing the expression of LDL receptor (LDLR), caveolin-1, cavin-1 and clathrin. (B) Quantitative analysis of the expression of proteins. " $\mathrm{P}<0.05$ vs. control; ${ }^{*} \mathrm{P}<0.05$ vs. Ang II.

is the crucial initiating event in early AS. LDLs are lipoprotein particles $20-30 \mathrm{~nm}$ in diameter and cannot pass through the intact endothelium via a paracellular pathway, but rather via the transporting process termed transcytosis. Importantly, accumulating evidence has pointed to the possible link between LDL transcytosis across ECs and the initiation of AS $(2,3,7)$.

Meanwhile, there is increasing evidence of the tight interactions between AS development and RAS activation. Of note, the crosstalk between dyslipidemia and RAS activation in atherogenesis has been emphasized. Ang II, as the principal effector molecule of RAS, is shown to be implicated in several important steps of AS development. However, whether or not Ang II can directly exert a pro-atherogenic effect by promoting LDL transcytosis across endothelial barriers, has not been defined. Previously, based on our newly established in vitro transcytosis model, our studies demonstrated that inflammatory factors CRP (9) and TNF- $\alpha$ (3) could increase caveolae-mediated LDL transcytosis across ECs and therefore promote LDL retention in human umbilical venous walls, as well as atherosclerotic lesion formation. Nevertheless, endogenous ceramide has also been shown to enhance the transcytosis of ox-LDL across ECs and promote the initiating step of AS, the subendothelial retention of lipids in the vascular wall (7). ROS have been emerging as essential intracellular secondary messengers and are also important signaling molecules known to increase endothelial permeability. Notably, we revealed that CRP indeed promoted a significant increase in ROS production in the ECs and DTT substantially decreased the CRP-stimulated upregulation of LDL transcytosis. Moreover, 
exogenous $\mathrm{H}_{2} \mathrm{O}_{2}$ was also found to cause an increase in LDL transcytosis in vitro (9), further supporting the involvement of ROS signaling in the CRP-stimulated increase in LDL transcytosis. In the present study, we aimed to ascertain whether or not Ang II is able to directly exert a pro-atherogenic effect by increasing LDL transcytosis across the endothelial barrier and promoting LDL retention in vascular walls.

With our in vitro transcytosis model, we firstly investigated the effects of Ang II on the transcytosis of LDL across ECs and found that Ang II significantly increased LDL transcytosis. Transcytosis inhibitor, $\mathrm{M} \beta \mathrm{CD}$, markedly prevented Ang II-stimulated LDL transcytosis. Of note, ROS inhibitor, DTT, also decreased LDL transcytosis induced by Ang II. To the best of our knowledge, this is the first study to ascertain that Ang II directly enhances LDL transcytosis across ECs. Such an effect may underlie the predictive value of Ang II in cardiovascular diseases. In the process of LDL transcytosis, LDL particles must be endocytosed into cells and then be transferred to the basolateral side and thus be exocytosed to the subendothelial space. During this process, an intermediate event of transcytosis occurs - LDL particles are taken up into the cytosol, but are not yet released (35). Therefore, determination of the intermediate form of LDL particles and the subendothelial retention of LDL can also reflect the activity of transcytosis. In the present study, we revealed that both the uptake of LDL in cells and the retention of LDL in human umbilical venous walls were largely elevated after Ang II exposure, which were consistent with the findings observed in the in vitro transcytosis model. But how does Ang II affect LDL transcytosis aross ECs?

Transcytosis is a complex multi-step process which includes endocytosis, intracellular trafficking and exocytosis. To date, clathrin-mediated endocytosis (CME) is the most widely studied endocytic pathway and plays critical roles in human health and disease. This pathway encompasses the ubiquitous uptake of nutrient-receptor complexes, adhesion molecules, growth factors, membrane transporters, toxin and viruses, the recycling of synaptic vesicles and activation of signaling pathways that regulate development and immune responses (36). Cargos delivered via the CME pathway are digested and degraded in the endolysosome ( $\mathrm{pH}, \sim 4.5)$ (37). Generally, receptor-mediated endocytosis via CME is for internal use. Specifically, researchers claim that LDLR binds LDL and is endocytosed in clathrin-coated pits via clathrindependent mechanisms. Acidification of early endocytic vesicles liberates LDL from the receptor and allows the cargo to be transported into lysosomes where LDL is degraded and cholesterol is salvaged for cellular use $(38,39)$.

Numerous studies have focused on transcytosis via caveolae. Caveolae are a particular type of lipid raft and have been described as $50-100 \mathrm{~nm}$, flask-shaped, non-clathrin-coated invaginations of the plasma membrane. A variety of receptors [e.g., LDLR, high-density lipoprotein receptor (HDL-R), albumin receptor (Alb-R), transferrin receptor (Tf-R), insulin receptor (Ins-R)], channels, and enzyme systems are located in caveolae microdomains (40). Accumulating evidence suggests that LDL transcytosis occurs mainly through a receptor-dependent pathway (41). Moreover, caveolae exhibit their highest frequency in ECs $(\sim 10,000 /$ cell). Since the population of clathrin-coated pits and vesicles is relatively small in ECs ( $\sim 3 \%$ of the total endothelial vesicles), caveolae play a major role in the process of transcytosis across the endothelium $(41,42)$.

Caveolin-1, an integral membrane protein $(20-22 \mathrm{kDa})$, is the major structural component of caveolae. Recently, evidence suggests that cavins, identified as a class of caveolae regulatory proteins, may also play an important role in caveolae formation. Data indicate a correlation between the levels of cavin-1 (polymerase transcript release factor, PTRF) and caveolin-1 and the abundance of caveolae. Notably, cavin-1 can be recruited by caveolins to plasma membrane caveolar domains and is necessary for caveolae formation $(43,44)$. In the present study, by determining the expression of these essential proteins involved in LDL transcytosis, including LDLR, caveolin-1, cavin-1 and clathrin, we observed that Ang II significantly upregulated the levels of LDLR, caveolin-1 and cavin-1, but Ang II exhibited no obvious effects on clathrin expression. These data provide compelling evidence proving that Ang II-induced LDL transcytosis across ECs is tightly associated with caveolaemediated transcytosis. More importantly, ROS inhibitor, DTT, significantly blocked the expression of proteins involved in LDL transcytosis mediated by caveolae.

Taken together, the present study for the first time demonstrated that Ang II exerted its pro-atherogenic effects by directly increasing LDL transcytosis across ECs and promoting LDL retention in vascular walls. Ang II-stimulated LDL retention in vascular walls appears to be a novel and key step in initiating AS. Mechanistically, proteins involved in caveolae-mediated transcytosis, including LDLR, caveolin-1 and cavin-1, were found to be tightly associated with Ang II-induced LDL transcytosis across ECs. However, this process was independent of clathrin in our study. Of note, ROS are critical factors in Ang II-induced LDL transcytosis. Hopefully, these findings will provide new insight into the crosstalk between dyslipidemia and RAS activation in atherogenesis, as well as novel strategies for the prevention or treatment of diseases related to AS.

\section{Acknowledgements}

We are grateful to the National Natural Science Foundation of China (no. 81503072 and no. 81373413) and the Hubei Province Health and Family Planning Scientific Research Project (no. WJ2015Q037).

\section{References}

1. Libby P, Ridker PM and Hansson GK: Progress and challenges in translating the biology of atherosclerosis. Nature 473: 317-325, 2011.

2. Bian F, Yang X, Zhou F, Wu PH, Xing S, Xu G, Li W, Chi J, Ouyang C, Zhang Y, et al: C-reactive protein promotes atherosclerosis by increasing LDL transcytosis across endothelial cells. Br J Pharmacol 171: 2671-2684, 2014.

3. Zhang Y, Yang X, Bian F, Wu P, Xing S, Xu G, Li W, Chi J, Ouyang $\mathrm{C}$, Zheng T, et al: TNF- $\alpha$ promotes early atherosclerosis by increasing transcytosis of LDL across endothelial cells: crosstalk between NF- $\kappa$ B and PPAR- $\gamma$. J Mol Cell Cardiol 72: 85-94, 2014.

4. Tabas I, Williams KJ and Borén J: Subendothelial lipoprotein retention as the initiating process in atherosclerosis: update and therapeutic implications. Circulation 116: 1832-1844, 2007.

5. Tuma $P$ and Hubbard AL: Transcytosis: crossing cellular barriers. Physiol Rev 83: 871-932, 2003. 
6. Bian F, Xiong B, Yang X and Jin S: Lipid rafts, ceramide and molecular transcytosis. Front Biosci (Landmark Ed) 21: 806-838, 2016.

7. Li W, Yang X, Xing S, Bian F, Yao W, Bai X, Zheng T, Wu G and Jin S: Endogenous ceramide contributes to the transcytosis of oxLDL across endothelial cells and promotes its subendothelial retention in vascular wall. Oxid Med Cell Longev 2014: 823071, 2014.

8. McAlister FA; Renin Angiotension System Modulator Meta-Analysis Investigators: Angiotensin-converting enzyme inhibitors or angiotensin receptor blockers are beneficial in normotensive atherosclerotic patients: a collaborative meta-analysis of randomized trials. Eur Heart J 33: 505-514, 2012.

9. Ohishi M, Dusting GJ, Fennessy PA, Mendelsohn FA, Li XC and Zhuo JL: Increased expression and co-localization of ACE, angiotensin II AT(1) receptors and inducible nitric oxide synthase in atherosclerotic human coronary arteries. Int J Physiol Pathophysiol Pharmacol 2: 111-124, 2010.

10. Schieffer B, Schieffer E, Hilfiker-Kleiner D, Hilfiker A, Kovanen PT, Kaartinen M, Nussberger J, Harringer W and Drexler H: Expression of angiotensin II and interleukin 6 in human coronary atherosclerotic plaques: potential implications for inflammation and plaque instability. Circulation 101: 1372-1378, 2000.

11. Daugherty A, Manning MW and Cassis LA: Angiotensin II promotes atherosclerotic lesions and aneurysms in apolipoprotein E-deficient mice. J Clin Invest 105: 1605-1612, 2000

12. Weiss D, Kools JJ and Taylor WR: Angiotensin II-induced hypertension accelerates the development of atherosclerosis in apoE-deficient mice. Circulation 103: 448-454, 2001.

13. Griendling KK, Minieri CA, Ollerenshaw JD and Alexander RW: Angiotensin II stimulates NADH and NADPH oxidase activity in cultured vascular smooth muscle cells. Circ Res 74 1141-1148, 1994.

14. Osterud B and Bjorklid E: Role of monocytes in atherogenesis. Physiol Rev 83: 1069-1112, 2003.

15. Pacurari M, Kafoury R, Tchounwou PB and Ndebele K: The Renin-Angiotensin-aldosterone system in vascular inflammation and remodeling. Int J Inflamm 2014: 689360, 2014.

16. Ni W, Kitamoto S, Ishibashi M, Usui M, Inoue S, Hiasa K, Zhao Q, Nishida K, Takeshita A and Egashira K: Monocyte chemoattractant protein-1 is an essential inflammatory mediator in angiotensin II-induced progression of established atherosclerosis in hypercholesterolemic mice. Arterioscler Thromb Vasc Biol 24: 534-539, 2004.

17. Li XC and Zhuo JL: Nuclear factor-kappaB as a hormonal intracellular signaling molecule: focus on angiotensin II-induced cardiovascular and renal injury. Curr Opin Nephrol Hypertens 17: 37-43, 2008.

18. Li W, Li Z, Chen Y, Li S, Lv Y, Zhou W, Liao M, Zhu F, Zhou Z, Cheng $\mathrm{X}$, et al: Autoantibodies targeting $\mathrm{AT}_{1}$ receptor from patients with acute coronary syndrome upregulate proinflammatory cytokines expression in endothelial cells involving NF-кB pathway. J Immunol Res 2014: 342693, 2014.

19. Rius C, Abu-Taha M, Hermenegildo C, Piqueras L, CerdaNicolas JM, Issekutz AC, Estañ L, Cortijo J, Morcillo EJ, Orallo F, et al: Trans- but not cis-resveratrol impairs angiotensin-II-mediated vascular inflammation through inhibition of NF- $\kappa \mathrm{B}$ activation and peroxisome proliferator-activated receptor-gamma upregulation. J Immunol 185: 3718-3727, 2010.

20. Rajagopalan S, Kurz S, Münzel T, Tarpey M, Freeman BA, Griendling KK and Harrison DG: Angiotensin II-mediated hypertension in the rat increases vascular superoxide production via membrane NADH/NADPH oxidase activation. Contribution to alterations of vasomotor tone. J Clin Invest 97: 1916-1923, 1996

21. Eto H, Miyata M, Shirasawa T, Akasaki Y, Hamada N, Nagaki A, Orihara K, Biro S and Tei C: The long-term effect of angiotensin II type 1a receptor deficiency on hypercholesterolemia-induced atherosclerosis. Hypertens Res 31: 1631-1642, 2008.

22. Daugherty A, Rateri DL, Lu H, Inagami T and Cassis LA: Hypercholesterolemia stimulates angiotensin peptide synthesis and contributes to atherosclerosis through the AT1A receptor. Circulation 110: 3849-3857, 2004.

23. Kunieda T, Minamino T, Nishi J, Tateno K, Oyama $T$, Katsuno T, Miyauchi H, Orimo M, Okada S, Takamura M, et al: Angiotensin II induces premature senescence of vascular smooth muscle cells and accelerates the development of atherosclerosis via a p21-dependent pathway. Circulation 114: 953-960, 2006.
24. Wang S, Subramanian V, Lu H, Howatt DA, Moorleghen JJ, Charnigo R, Cassis LA and Daugherty A: Deficiency of receptor-associated protein attenuates angiotensin II-induced atherosclerosis in hypercholesterolemic mice without influencing abdominal aortic aneurysms. Atherosclerosis 220: 375-380, 2012.

25. Wu SJ, Soulez M, Yang YH, Chu CS, Shih SC, Hébert MJ, Kuo MC and Hsieh YJ: Local augmented angiotensinogen secreted from apoptotic vascular endothelial cells is a vital mediator of vascular remodelling. PLoS One 10: e0132583, 2015.

26. Libby P: Inflammation in atherosclerosis. Arterioscler Thromb Vasc Biol 32: 2045-2051, 2012.

27. Schupp N, Kolkhof P, Queisser N, Gärtner S, Schmid U, Kretschmer A, Hartmann E, Oli RG, Schäfer S and Stopper H: Mineralocorticoid receptor-mediated DNA damage in kidneys of DOCA-salt hypertensive rats. FASEB J 25: 968-978, 2011.

28. Calhoun DA, White WB, Krum H, Guo W, Bermann G, Trapani A, Lefkowitz MP and Ménard J: Effects of a novel aldosterone synthase inhibitor for treatment of primary hypertension: results of a randomized, double-blind, placebo- and active-controlled phase 2 trial. Circulation 124: 1945-1955, 2011.

29. Takata H, Yamada H, Kawahito H, Kishida S, Irie D, Kato T, Wakana N, Miyagawa S, Fukui K and Matsubara H: Vascular angiotensin II type 2 receptor attenuates atherosclerosis via a kinin/NO-dependent mechanism. J Renin Angiotensin Aldosterone Syst 16: 311-320, 2015.

30. Catar RA, Müller G, Heidler J, Schmitz G, Bornstein SR and Morawietz H: Low-density lipoproteins induce the renin-angiotensin system and their receptors in human endothelial cells. Horm Metab Res 39: 801-805, 2007.

31. Wang X, Phillips MI and Mehta JL: LOX-1 and angiotensin receptors, and their interplay. Cardiovasc Drugs Ther 25: 401-417, 2011.

32. Sendra J, Llorente-Cortés V, Costales P, Huesca-Gómez C and Badimon L: Angiotensin II upregulates LDL receptor-related protein (LRP1) expression in the vascular wall: a new pro-atherogenic mechanism of hypertension. Cardiovasc Res 78: 581-589, 2008.

33. Cardona-Sanclemente LE, Medina R and Born GV: Effect of increasing doses of angiotensin II infused into normal and hypertensive Wistar rats on low density lipoprotein and fibrinogen uptake by aortic walls. Proc Natl Acad Sci USA 91: 3285-3288, 1994.

34. Keidar S, Heinrich R, Kaplan M, Hayek T and Aviram M: Angiotensin II administration to atherosclerotic mice increases macrophage uptake of oxidized ldl: a possible role for interleukin-6. Arterioscler Thromb Vasc Biol 21: 1464-1469, 2001.

35. Frank PG, Pavlides S and Lisanti MP: Caveolae and transcytosis in endothelial cells: role in atherosclerosis. Cell Tissue Res 335: 41-47, 2009.

36. Kirchhausen T, Owen D and Harrison SC: Molecular structure, function, and dynamics of clathrin-mediated membrane traffic. Cold Spring Harb Perspect Biol 6: a016725, 2014.

37. El-Sayed A and Harashima H: Endocytosis of gene delivery vectors: from clathrin-dependent to lipid raft-mediated endocytosis. Mol Ther 21: 1118-1130, 2013.

38. Sorrentino V, Nelson JK, Maspero E, Marques AR, Scheer L, Polo $S$ and Zelcer N: The LXR-IDOL axis defines a clathrin-, caveolae-, and dynamin-independent endocytic route for LDLR internalization and lysosomal degradation. J Lipid Res 54: 2174-2184, 2013.

39. Howes MT, Mayor S and Parton RG: Molecules, mechanisms, and cellular roles of clathrin-independent endocytosis. Curr Opin Cell Biol 22: 519-527, 2010

40. Simionescu M, Popov D and Sima A: Endothelial transcytosis in health and disease. Cell Tissue Res 335: 27-40, 2009.

41. Pavlides S, Gutierrez-Pajares JL, Iturrieta J, Lisanti MP and Frank PG: Endothelial caveolin-1 plays a major role in the development of atherosclerosis. Cell Tissue Res 356: 147-157, 2014.

42. Predescu SA, Predescu DN and Malik AB: Molecular determinants of endothelial transcytosis and their role in endothelial permeability. Am J Physiol Lung Cell Mol Physiol 293: L823-L842, 2007.

43. Nassar ZD, Hill MM, Parton RG and Parat MO: Caveola-forming proteins caveolin-1 and PTRF in prostate cancer. Nat Rev Urol 10: 529-536, 2013.

44. Regazzetti C, Dumas K, Lacas-Gervais S, Pastor F, Peraldi P, Bonnafous S, Dugail I, Le Lay S, Valet P, Le Marchand-Brustel Y, et al: Hypoxia inhibits cavin-1 and cavin-2 expression and down-regulates caveolae in adipocytes. Endocrinology 156: 789-801, 2015. 